\title{
Políticas en la agricultura familiar, Cañete - 2018
}

\section{Policies in family agriculture, Cañete -2018}

\author{
Bessy Castillo Santa María \\ Universidad Nacional de Cañete, Perú \\ Irma Milagros Carhuancho Mendoza \\ https://orcid.org/0000-0002-4060-5667 \\ Universidad Cesar Vallejo, Perú \\ Rubén Andrés Moreno Sotomayor \\ Instituto de Educación Superior Tecnológico Publico “Cañete”, Perú
}

Autor por correspondencia: bcastillosm@hotmail.com; irmamilagros3@gmail.com; rubenchi77@yahoo.com.ar

Fecha de recepción: 22 de septiembre del 2019 - Fecha de aceptación: 05 de diciembre del 2019

\section{Resumen}

El objetivo de la presente investigación es determinar si las políticas influyen en la agricultura familiar de subsistencia y consolidación, en Cañete. Estudio que se desarrolló mediante enfoque cualitativo, con entrevista a profundidad, mediante una guía de preguntas y como unidad de análisis dirigentes agrarios entre hombres y mujeres; autoridades de instituciones del sector agrario, que suman seis; empleando para el análisis el programa Atlas ti, para la triangulación. La agricultura de subsistencia es limitada en el desarrollo del campo, por la carencia de los recursos económicos y proyectos institucionales que contribuyan a mejorar la calidad de vida de las familias que se dedican a la agricultura familiar, la agricultura consolidada cuenta con capacidad de generar sus propios recursos, se apoya en la tecnología para la producción en gran escala, en concordancia con las políticas públicas nacionales e internacionales. Existe desconocimiento de la ley, tanto de autoridades y productores donde la agricultura de subsistencia en el valle de Cañete, esta privada de recursos económicos, y tecnológicos, efecto de ello solo produce para el autoconsumo, en tanto que la agricultura consolidada desarrolla el campo con cultivos de agro exportación generadora de divisas y fuente de trabajo.

Palabras claves: políticas; agricultura; subsistencia; consolidada

\footnotetext{
Abstract

The objective of this research is to determine if policies influence family subsistence and consolidation agriculture in Cañete. Study that was developed through a qualitative approach, with an in-depth interview, through a guide of questions and as a unit of analysis agrarian leaders between men and women; authorities of agricultural sector institutions, totaling six; using the Atlas ti program for analysis, for triangulation. Subsistence agriculture is limited in the
} 
development of the countryside, due to the lack of economic resources and institutional projects that contribute to improving the quality of life of families engaged in family farming, consolidated agriculture has the capacity to generate its own resources, relies on technology for large-scale production, in accordance with national and international public policies. There is ignorance of the law, both of authorities and producers where subsistence agriculture in the Cañete valley, is deprived of economic and technological resources, the effect of this only produces for self-consumption, while consolidated agriculture develops the field with agro export crops generating foreign exchange and source of work.

Key words: policies; farming; subsistence; consolidated

\section{Introducción}

En el Perú la agricultura familiar (AF) esta diferenciada por su vulnerabilidad agroecológica en las tres regiones naturales. Cañete está ubicada en la costa peruana, una ciudad que se caracteriza por la agricultura, sin embargo, no es ajena a la inoperancia de las políticas púbicas en el sector agrario, los agricultores optan esta actividad por convicción y subsistencia. En el valle de Cañete, no existe funcionabilidad de las políticas en favor de la AF, que se encuentran asentadas en las zonas rurales, donde cada agricultor focaliza su trabajo en forma particular y familiar, destacando la necesidad de generar recursos para su sobrevivencia, acuden a trabajos como obreros en las actividades de una agricultura consolidada que se dedica a las agro exportaciones, logrando así mantener su vida diaria.

La presente investigación se basa en determinar si las políticas públicas influyen en la agricultura familiar en Cañete- 2018, respecto a la agricultura de subsistencia y la agricultura consolidada, marcada diferencia de dos grupos diferentes el primero carente de atención de entidades gubernamentales, con escasos recursos e insipiente tecnología, la segunda enmarcada a un sistema de calidad internacional con cultivos de agro exportación, quienes trabajan directamente con el gobierno, planteando leyes en beneficio propio, contribuye a generar mano de obra calificada y no calificada.

Sin embargo, estés dos sistemas agrarios generan, la principal actividad económica productiva en el valle de Cañete, por ello la necesidad de conocer como las políticas nacionales agrarias favorecen principalmente a la $\mathrm{AF}$, que vienen realizando la venta de sus predios por el crecimiento poblacional para las habilitaciones urbanas, a través del cambio de uso del suelo.

Las Políticas, según el Banco Interamericano de Desarrollo (Banco Interamericano de Desarrollo, 2011), busca el perfeccionamiento de las políticas públicas enmarcadas en diferentes factores como estabilidad, así como también la adaptabilidad por lo que permite satisfacer el interés en el ámbito público.

Sin embargo Torres \& Santander (2013), afirman que las políticas públicas vienen a ser el resultado de diversas acciones desarrolladas en forma colectiva en la sociedad, a través de diferentes servicios que presta el gobierno en el ámbito de su accionar, con funciones variadas, lo que impide accionar al estado en una sola política, por lo que permite garantizar múltiples coordinaciones inter institucionales tanto en el sector público así como también privado para el logro de los objetivos planteados. En tanto que las políticas públicas son por tanto una acción 
que está relacionada con el flujo de información preferentemente a un objetivo público; respecto a la finalidad o mediante un propósito que están determinadas, a lograr unificar la información dispersa en los diferentes ministerios (Frei, 1997, p. 162). Frente a ello la necesidad de mejorar el desarrollo de capacidades mediante la innovación y la mejora de conocimiento en las personas, los dirigentes, los líderes de las organizaciones y la sociedad, conllevan un cambio guiado y sostenido de capacidades, (Programa de las Naciones Unidas para el Desarrollo; PNUD, 2009).

Las políticas internacionales propuestas en el Objetivo de Desarrollo del Milenio (ODM), enmarca en el Plan Bicentenario El Perú hacia el 2021, inmersas en las Políticas de Estado del Acuerdo Nacional, tomando como base el Minagri quien plantea la Política Nacional Agraria, para el trabajo conjunto con los gobiernos regionales, que estos a su vez implementa el Plan de Desarrollo Regional, direccionando acciones de trabajo a desarrollar con los gobiernos locales en bien de los actores de las actividades agrícolas a pequeña escala, integrante de la agricultura familiar, tal como se muestra en la figura 1. Lo que demuestra el incumplimiento de las políticas establecidas en los diferentes estratos de gobierno.
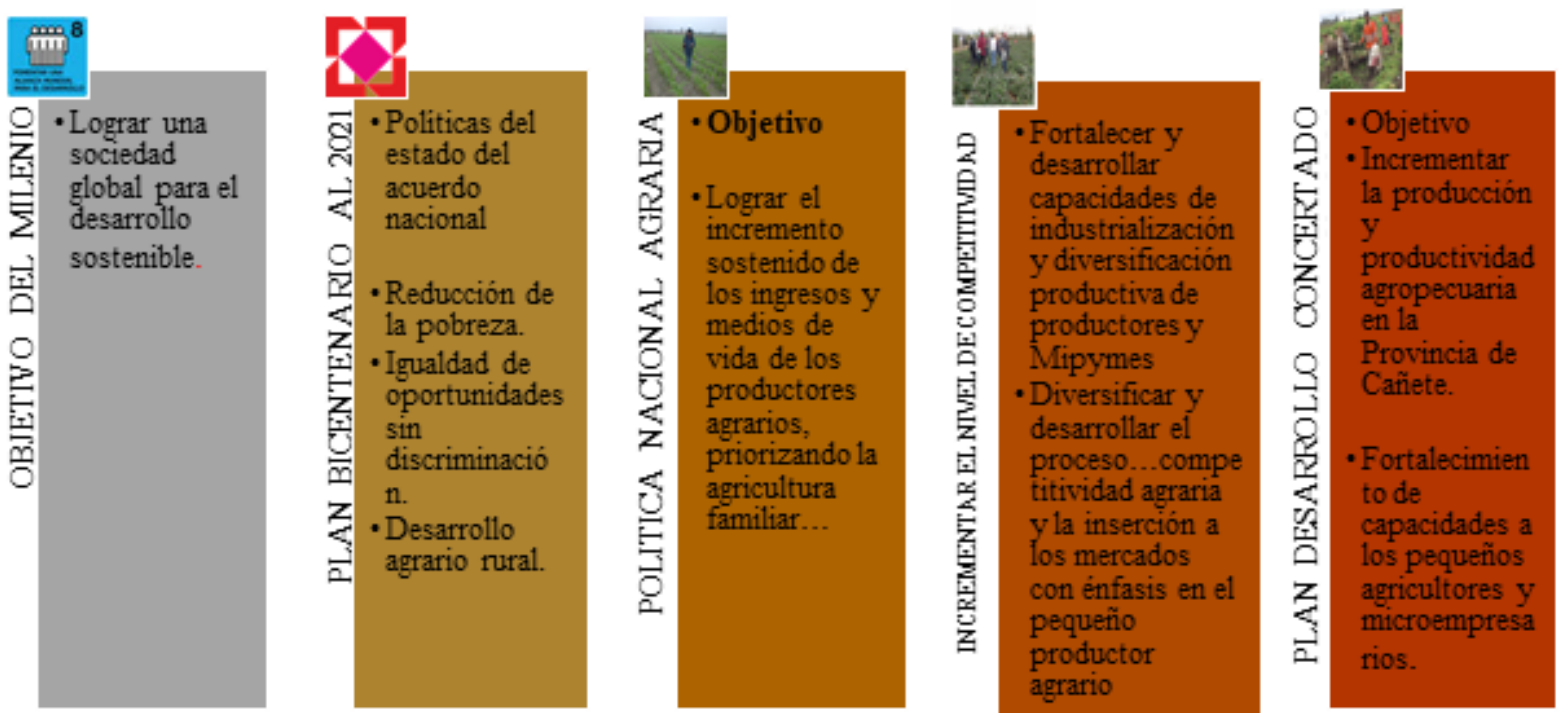

Figura 1. Secuencia lógica de la política internacional, nacional, regional y local

Fuente: elaboración propia: FAO, Centro Nacional de Planeamiento Estratégico (Centro Nacional de Planeamiento Estratégico, 2011) Centro Nacional de Planeamiento Estratégico, Minagri, Plan de Desarrollo Regional Concertado actualizado 2016-2021 del Departamento de Lima, Plan de Desarrollo Concertado de Cañete 2008-2021

El Estado peruano focaliza doce políticas públicas en la agricultura, tal como se muestra en la figura 2. Desarrolladas mediante reuniones entre múltiples instituciones del sector agrario, expertos, colegio de profesionales, organizaciones de productores, empresas, entre otros (Minagri, 2016), las cuales tiene vigencia actual. 


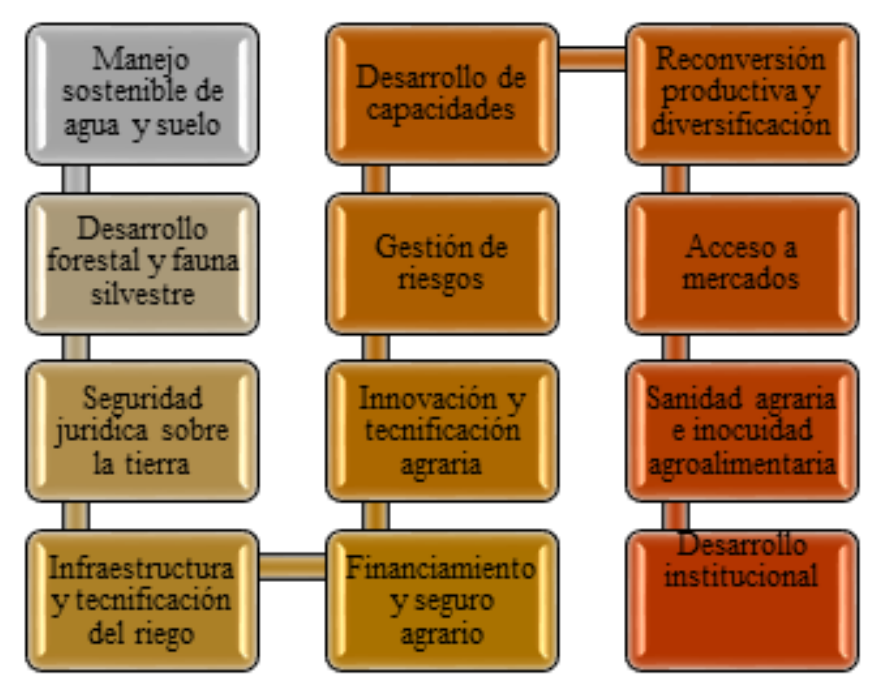

Figura 2. Política nacional agraria PNA

Fuente: elaborado por el Minagri 2016

Para efecto de una diferenciaciòn entre la agricultura familiar y la agricultura consolidada existente en el valle de Cañete, se presenta la tabla 1, que refleja su accionar, constante frente a una polìtica sin estrategía a los menos favorecidos por parte de los gobiernos tanto nacional, regional y local. Siendo de gran importancia de los procesos organizacionales y de los apoyos públicos y privados... del trabajo colectivo consolidado y de las políticas pública (Sabourin, Aveline, Petersen, \& Para, 2018).

Según Monterrey (2017) reporta que el Perú es el país que cuenta con mayor cantidad de productores dedicados a la AF, el Estado destina en promedio de 3,76\% del presupuesto nacional, para la actividad productiva. No obstante Salcedo \& Guzmán (2014) indican que la AF enfrenta el desafio de la heterogenidad de situaciones en cuanto a la carencia de recursos económicos, asistencia dirigida y circunstancias propias de la agricultura. "Las políticas públicas de desarrollo territorial rural, consideran que la AF es una mezcla que presenta una intensidad distinta en los países en función de factores endógenos" (Balestro, Sayago, Valencia, \& Sabourin, 2019, p. 51), en consecuencia "las politicas públicas no siguen recetas, por lo que no pueden ser considerados procesos amorfos, desconectados de la realidad social" (Flexor \& Grisa, 2016, p. 51).

Las políticas públicas al tiempo de reafirmar la presencia, permanencia e importancia de la $\mathrm{AF}$ en los territorios rurales y en el desarrollo de los países, buscan integrar un abordaje más complejo que el rubro productivo, propio de las políticas agrícolas de naturaleza homògenea (Ec (Echeverri P. \& Renault A., 2017, p.17).

Los diferentes desáfios que enfrenta la AF son la heterogenidad, por lo que es necesario iniciar con las "matrices de política públicas" que permitan avanzar en las mejoras de la asociatividad y asegurar el autoconsumo familiar (Ramos, 2016).

Por ello las posibilidades y estrategias se debe avanzar con las políticas públicas enfocados a los sectores desfavorecidos del agro, donde a la AF, se les proporcione elementos 
para resistir a las formas de desposesión frente a la permanencia de gobiernos conservadores (Villarreal, 2018).

En tal sentido la Organizaciòn de las Naciones Unidas para la Alimentaciòn y la Agricultura (FAO, 2016) reporta que el 70\% de los pobres del mundo viven en las zonas rurales, la agricultura es la principal fuente de ingresos y de trabajo. El Ministro de Agricultura y Riego (Minagri, 2015) consideró que en el Perú el desarrollo sostenible de la AF representa el 97\% del total nacional, existiendo más de 2.2 millones de Unidades Agropecuarias (UA) en distintas regiones del país.

Tabla. 1

Efectos de una agricultura AF y una $A C$.

AGRIULTURA FAMILIAR (AF) $\quad$ AGRICULTURA

CONSOLIDADA (AC)

El manejo sostenible de agua y suelo insipiente por no contar con

En gran mayoria desarrollan la parametros establecidos de calidad, y supervisiòn de las instituciones responsables, lo que provoca desperdicio de agua, formaciòn de suelos presevacion del agua y suelo, a traves salitrosos y compactos.

Desarrollo de flora y fauna silvestre la existencia de trabajar la totalidad de su predio, carecen de flora y fauna.

de riego por goteo.

Seguridad juridica sobre la tierra genera desconfianza entre los propietarios que no logran culminar su formalizaciòn, ante el gobierno.

Infraestructura y tecnificaciòn de riego, ausentes en los campos de cultivos.

Fortalecimiento y seguro agrario, carecen sobre efectos naturales, pero si cuentan con seguro agrario de salud.

Financiamiento y seguro agrario no repercute en el ambito del valle por el estado, si por financieras privadas, el seguro implementado para la salud del agricultor mas no por desastres naturales.

La innovaciòn y tecnificaciòn agraria estan limitados.

Gestiòn de riesgo, desconocimiento en su aplicaciòn.

de la entidad gubernamental, si de la Desarrollo de capacidades ausentes privada logrando beneficio propio.

Reconversiòn productiva y diversificaciòn, fracaso de programas.

El desarrollo de capaciades ausentes para la AF, si por las empresas privadas comercializadoras de insumos quìmicos

Acceso a mercado, nulo

Sanidad agraria e inocuidad agroalimentaria, preservan por iniciativa propia.

Sanidad agraria ausente en la AF

Desarrollo institucional desarticulado
En la flora preservan su conservaciòn por estandares de calidad. Y la fauna restringuida.

Cuenta con documentos reglamentarios.

Cuenta con servicios de instalaciòn tecnificada dentro de sus predios.

Cumplen en funciòn de desarrollo del personal y empresarial.

Se mantiene según su hegemonìa econòmica

Desarrollan investigaciòn e invierten en tecnologìa.

Sistemas implementadosl

Genera continuo desarrollo en su personal.

Interes segùn mercado agroexportador.

Capacitaciòn constante.

Interacciòn internacional.

Preservan por intereses de mercado mundial.

Polìticas de gobierno aplicables. Fortalecidos.

La segunda categoría: la agricultura familiar, se focaliza mediante el padrón de socios obtenidos de la Junta de Usuarios de productores dedicados a la AF, distribuidas en sus siete (7) comisiones de regantes, cada uno de ellas desarrolla actividades en bien de sus usuarios. Logrando identificar que de 23,369.4 Has. Agrícolas existentes en el valle de Cañete, solo 2228 ha., se dedican a la AF con menos de 2 has., tal como se detalla en la tabla 2. 
Tabla. 2

Distribución de áreas agrícolas dedicadas a la $A F$, por comisiones de regantes, en el valle de Cañete, Lima Provincias. (2018)

\begin{tabular}{lll}
\hline No. & Comisiones de Regantes del Sub distrito de Riego Cañete & $\begin{array}{l}\text { Agricultores } \\
>0.5 \mathrm{a}<2 \text { Has. }\end{array}$ \\
\hline 1 & C. Pachacamilla & 96 \\
2 & C. Nuevo Imperial & 1064 \\
3 & C. María Angola & 111 \\
4 & C. Palo Herbay & 218 \\
5 & C. San Miguel & 253 \\
6 & C. Viejo Imperial & 354 \\
7 & C. Huanca & 132 \\
\hline \multicolumn{2}{c}{ Total } & $\mathbf{2 2 2 8}$ \\
\hline
\end{tabular}

Fuente: Padrón de socios de la Junta de Usuarios 2018

Sin embargo, Maletta (2017) sostiene que las categorías de escala de las pequeñas unidades familiares (PAF) son consideradas en hectáreas de riego en costa (HRC) y determina los rangos en: unidades PAF de (menor de 0,5 HRC), unidades PAF de subsistencia (0,5 a menos de 2 HRC), unidades PAF intermedias (2 a menos de 5 HRC) y finalmente las unidades de PAF excedentarias (5 a menos de $10 \mathrm{HRC}$ ).

Minagri (2015) indica que la agricultura familiar es una cualidad de vida y revaloriza la producción que practican tanto hombres y mujeres del contexto familiar que viven en un territorio de ámbito rural, en el que tienen un compromiso variado en la producción a través de sistemas, que impulsan íntimamente en la unidad productiva familiar, dedicados a la producción agrícola, como la pecuaria, igualmente el manejo forestal, seguido de la industrial rural, la pesca artesanal, acuícola y apícola, y otros. Tal como se expresa en la figura 3. Donde la innovación es el resultado de un proceso intensivo de generación de ideas que permiten encontrar nuevas y mejores soluciones a las existentes, frente a la necesidad de resolver un problema (Ramìrez, Ruilova \& Garzòn, 2015).

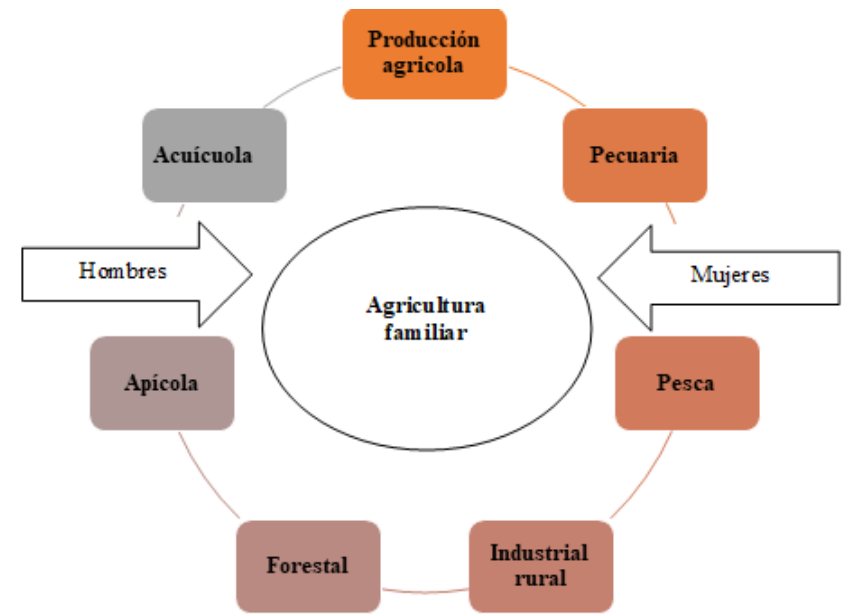

Figura 3. Sistemas de producción en la agricultura familiar Fuente: elaboración propia. 
La agricultura familiar es considerada en el mundo occidental como un fenómeno social que se complica por razones impuestas por la burocracia y la industria que están más posesionada con la sociedad, creen que la agricultura familiar está desfasada y antigua, sin embargo, precisan que es la más rica porque revalora la vida en familia destacando que es aceptable y seductora porque la gente cultiva y vive, optando una forma de vida armoniosa (Ploeg, 2013, p. 6). En consecuencia la AF en el valle de Cañete, los trabajos de campo lo realizan en forma manual, logrando mantener la sobrevivencia mediante su propia tecnologia, y a razon de sus concidiones de vida de subsistencia en largos periodos de tiempo, efectivizando su contribuciòn con el medio ambiente, al no emplear maquinarias pesadas que compactan el suelo, y agudizen el remplazo de la mano de obra de la gente del campo, y por consiguiente evitan el uso de combustibles de residuos fòsiles.

En la presente investigación se identifica dos subcategorías que se muestra en la figura 4, que corresponden a la agricultura familiar denominada agricultura de subsistencia y agricultura consolidada.

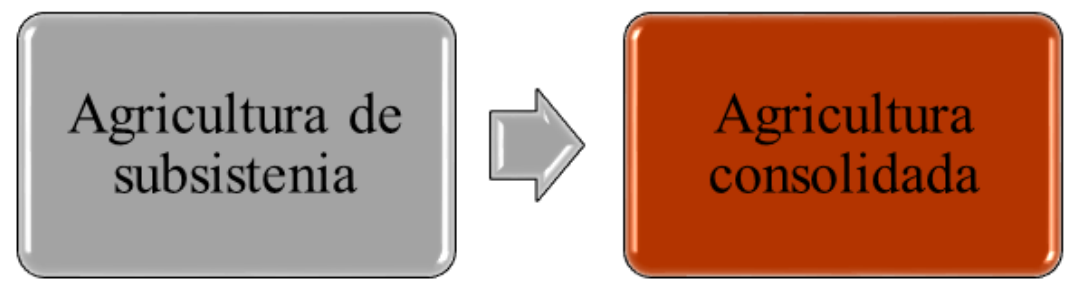

Figura 4. Sub categoría de la agricultura familiar.

Fuente: elaboración propia

Mejorar la pequeña agricultura para combatir la pobreza rural alimentaria mediante nuevos programas con instituciones ligadas al sector, que impulsan el desarrollo agrario frente al cambio climático y lograr habilidades con distintas perspectivas transitoria, a fin de mejorar los recursos económicos de las familias por el estado, direccionando en beneficio de las zonas rurales mas necesitadas (Quintero, 2017). Por consiguiente considerar que la AF esta en emergencia por el accionar de las autoridades en los distintos niveles de gobierno, por efecto de políticas dividas en los diferentes estratos de gobierno que perjudican el accionar y la estabilidad del agro que determina un accionar totalmente problemático, manifestandose en discusiones, construcciones e implementación de nuevas perspectivas en la politica para la AF (Gónzales, 2016). Por lo que implica la necesidad urgente de enfocar con mayor severidad el cumplimiento de las polìticas pùblicas en el agro peruano y latino ameriano de zonas desprotegidas, que trabajan al olvido del gobierno.

\section{Metodología}

El estudio se realizó en base al paradigma interpretativo. Calificado como interpretativo figurado naturalista, humanista y fenomenológico (Rico, 2006), con un enfoque cualitativo, tipo exploratorio, donde (Troncoso-Pantoja \& Amaya-Placencia, 2016) consideran la técnica entrevista como una gran herramienta para obtener datos valiosos. Y empleando como instrumento la guía de entrevista abierta que nos permite seguir la secuencia lógica de las preguntas realizado a tres dirigentes y tres funcionarios de las instituciones agrarias más 
representativas del valle de Cañete, formulado en forma individual a cada entrevistado en un tiempo de 30 minutos, en diferentes días, cuyos datos obtenidos se procesó mediante el programa de ATLAS ti, para la triangulación de las respuestas alcanzadas. Las categorías en estudio se basan al conocimiento in situ de la realidad existente.

\section{Resultados}

En el Perú, el gobierno de turno diseña sus propias políticas las mismas que son modificadas por el siguiente gobierno es decir no existe una política de estado continua y sólida con proyección de crecimiento. Las políticas públicas en la AF está siempre inoperativa por carecer de instrumentos necesarios para impulsar su accionar es así referente a la: Gestión del agua, proponen plantear alternativas para su uso más eficiente del recurso hídrico, en vista que el manejo de agua en Cañete se realiza mediante el sistema de gravedad, siendo los pequeños productores que no cuentan con infraestructura tecnificada, provocando erosión, escorrentía en la aplicación de fertilizantes, desperdicio de agua, salinización de los suelos, escasa producción, por el mal uso en la gestión de agua y suelo. Los moradores de la cuenca alta desconocen de tecnificación, por lo que sugiere plantear alternativas para el uso eficiente. El manejo del recurso se desarrolla por mitas, con disponibilidad de agua de siete a quince días, permitiendo regular su entrega entre los usuarios, las comisiones de regantes a través del sectorista distribuyen el recurso hídrico, dando cuenta posteriormente a la junta de usuarios. "El manejo racional de los recursos hídricos contribuye al mejoramiento de la calidad de vida del hombre" (Luna \& Maldroñero, 2016, p. 12)

Del mismo modo, la disponibilidad hídrica en el valle, es permanente para uso agrícola sin restricciones salvo excepciones por efectos ambientales, formas reguladas para su mantenimiento y conservación establecida en forma conjunta entre los usuarios organizados a través de la Junta de Usuarios, para una eficiente distribución del recurso agua. Por otra parte, la carencia de un protocolo de prevención por efecto de desastres naturales en las cuencas de los ríos es inesperada o agresiva, en épocas de crecientes y su implementación permitiría prevenir, reducir los riesgos por efectos de fenómenos naturales del rio Cañete. Solo los canales principales cuentan con infraestructura técnica y los canales laterales sin infraestructura, que funcionan en condiciones precarias. La gestión del suelo, inapropiados por el mal uso del mismo, carente de nutrientes, de contenido de materia orgánica y de apoyo en asesoramiento técnico. Es necesario resaltar que el valle de Cañete cuenta con recursos hídricos durante todo el año.

Respecto al desarrollo forestal y fauna silvestre, la importancia radica en el ordenamiento territorial, y la sostenibilidad de los recursos, con limitadas áreas de conservación, debido a que políticas de protección existentes no son tomadas en cuenta por las autoridades competentes. Permitiendo la extinción de la fauna silvestre por el uso excesivo e indiscriminado de pesticidas, basados en una agricultura sin restricción de productos tóxicos. Sin embargo, la pequeña agricultura es la que da la sostenibilidad ambiental con trabajos propios de su actividad productiva, y la agricultura consolidada, ligados a la agricultura agro exportadora desarrolla la conservación, preservación de la flora y fauna en los límites de su propiedad mas no en sus campos de cultivo. Generando limitadamente el restablecimiento de un microclima para la flora y fauna silvestre. Sin embargo, la flora y fauna silvestre a orilla de los ríos están resguardados para evitar desborde del río, erosión, protegiendo la conservación de los recursos 
hidrobiológicos, y la biodiversidad, que están regulada y reglamentada por el Servicios Nacional Forestal y de Fauna Silvestre - Serfor.

La seguridad jurídica sobre la tierra, está relacionada con tres principales factores como: la titulación de la propiedad agraria, donde la mayoría de los agricultores no cuentan con título de propiedad de sus predios, más aún inscritos en registros públicos, siendo fundamental considerar para efectos de legalidad, y lograr que cada propietario cuente con el documento de titulación. Segundo factor, saneamiento físico legal, que acredite su propiedad, para lo cual solo cuentan con la resolución emitida durante la reforma agraria. Lo que deriva el desinterés del gobierno regional para sanear los predios agrícolas hasta culminar la titulación. El tercer factor es de formalización, lo cual impide la titulación de la propiedad agraria, riesgo que genera desconfianza en los agricultores. A efecto la agencia agraria de ámbito local, emite el certificado posición, para mantener sus propiedades a buen recaudo, mas no legalmente ante la institución competente para su inscripción. Al no contar con el título de propiedad impide un abanico de oportunidades como: créditos agrarios, informalidad, baja producción, incipiente comercialización, abandono del trabajo agrícola, venta de sus terrenos y cambio de uso de suelo.

En el marco de la infraestructura y tecnificación del riego, existe una baja calidad en la distribución del agua, debido a construcciones que están en mal estado que va desde los canales principales, continúa por canales secundarios y por último hasta los laterales que van directo a los predios de los agricultores. Dificultando una distribución correcta y equitativa del recurso agua, lo que provoca ausencia de riego en campos agrícolas ubicados en zonas alejadas, o provocando inundaciones, lo que genera insatisfacción en los usuarios que se valen del servicio para el mantenimiento de sus cultivos. La carencia de políticas en la infraestructura y tecnificación de riego, para los productos agrarios, impide el mejoramiento de la producción y organización de sus cultivos, siendo una alternativa el uso eficiente de los recursos hídricos.

El desarrollo de capacidades es restringido por el sector gubernamental, para productores individuales, existiendo iniciativas por parte de la actividad privada. Sin embargo, instituciones gubernamentales como el Servicio Nacional de Sanidad Agraria (Senasa), Agencia Agraria y la Autoridad Local del Agua (Ala), vienen trabajando exclusivamente con los grupos organizados como: cooperativas, asociaciones, pequeñas, medianas y grandes empresas en temas de sanidad agraria, con escasos recursos y logística limitada que impide que se abarque a una mayor población agrícola de la AF.

En la gestión de riesgo, se presenta una débil prevención, factor de peligro que suma canales en mal estado, con malezas, acumulación de envases de agroquímicos, sin revestimiento, obstrucciones que impide la fluidez del recurso agua. Sin embargo, las consideraciones a tomar en cuenta no están orientadas, a prevenir posibles desastres a consecuencia de fenómenos naturales, en vista que la infraestructura está en mal estado, y la tecnificación es mínima perjudicando a los suelos erosionados, salinos, infértiles, en perjuicio de los productores. En tanto, los riesgos más frecuentes se presentan en personas asentadas a orillas de los ríos, que dan uso a zonas protegidas de los márgenes establecidos por ley, para la construcción de sus viviendas, ampliación de áreas de cultivos, que está altamente prohibido. Demostrando débil gestión de las instituciones competentes. Efecto de ello, las aguas de los ríos en época de avenida afecten con desbordes e inundaciones, acto que inicia con las coordinaciones entre instituciones

Esta obra se comparte bajo la licencia Creative Common Atribución-No Comercial 4.0 International (CC BY-NC 4.0) Revista de la Universidad Internacional del Ecuador. URL: https://www.uide.edu.ec/ 
involucradas del recurso agua, para su mantenimiento en la prevención, permitiendo la reducción del riesgo momentáneamente sin planificar las consecuencias futuras.

En la innovación y la tecnificación agraria en el valle de Cañete, se necesita accionar en base a los aspectos volubles del cambio climático que perjudica a los productores que no están preparados y no cuentan con recursos para continuar su trabajo, toda vez que estos fueron arrasados por inundaciones perdiendo totalmente su cultivo, a ello se suma el desinterés de las instituciones públicas, como las municipalidades y la región que desarrollan trabajos individualmente y aislados sin tomar en cuenta a los damnificados de las zonas críticas. En tanto, que la investigación y la tecnificación carecen de importancia en el valle por las instituciones responsables para mejorar la producción y productividad, esto refleja que los recursos genéticos no cuentan con la protección del Estado y menos aún por la gran mayoría de productores. Siendo los agro exportadores consiente de su actividad productiva continúan en la innovación constante de su tecnología a fin de mantener la competitividad a nivel internacional y redoblan esfuerzos para su continuidad en el mercado.

En el ámbito del financiamiento y seguro agrario, es importante resaltar que el sector público y al sector privado brindan financiamiento, pero con mayor acogida del segundo, en vista de una atención oportuna de los créditos agrarios, en consecuencia, la desaparición de la entidad financiera pública es un antecedente de una débil gestión, en las políticas implementadas, no acorde a la realidad existente, cuyos recursos no son focalizados a intereses de una AF. Por consiguiente, el financiamiento privado, es veraz, oportuno y con mayor acogida en la agricultura, con una gran desventaja por sus altos intereses en la entrega de sus créditos. Respecto al seguro agrario, en el valle de Cañete, por condiciones climáticas por primera vez se ejecutó un bono de financiamiento a los productores que cumplieron en presentar documentos que acredite su propiedad y su cultivo.

Reconversión productiva, no se desarrolló adecuadamente por carecer el programa del Estado con objetivo claros, direccionando solo a grupos organizados, programa sin planteamiento y promoción que facilite al agricultor obtener bondades propias de su trabajo. Ejemplo de ello es que los pequeños agricultores dedicados a la agricultura familiar con la siembra de algodón, participaron de la reconversión productiva dejando la siembra de su cultivo inicial, para continuar y mejorar sus ingresos con el cultivo de quinua, con gran auge e impacto a nivel internacional. Este programa fracaso y dejó disconforme y pérdidas en los agricultores, dada la escasa visibilidad de este cultivo en la costa peruana, oriunda de los andes, lo que provocó que toda la producción sea rechazada por restos de plaguicidas en las cosechas y bajas en el precio del mercado nacional. Contribuyendo a que estos agricultores generen deudas impagables al Estado, que viene trabajando sin orientación y planificación en favor de la AF. Oportunismo de programas que el gobierno implementa para aplacar el rechazo los agricultores de la AF.

El acceso al mercado, es limitado directamente para los de la AF, siendo los intermediarios los principales actores de la comercialización y ganancias, afectando la siembra y colocación de sus productos, con restringidas mejoras en sus campos y su familia, logrando que la AF, continúe postergada a consecuencia de una débil gestión y aplicación de la ley. Que tiene 
como objetivo establecer las responsabilidades del Estado en la promoción y desarrollo de la AF. (Minagri, 2015)

La sanidad agraria, implementada para mejorar las condiciones de los productos agrícolas en el campo, mediante la erradicación de enfermedades a través de la institución competente como Senasa, que cumple un rol limitado de funciones, por lo que carecen de recursos y personal técnico indispensable para capacitaciones en los diversos lugares de su jurisdicción. Por tanto, esto viene provocando consecuencias irremediables por el uso irresponsable de productos tóxicos, contaminación de los campos agrícolas, cosechas, suelos, aguas, aire y la comunidad en general quienes consumen directamente a través de la venta en los mercados, sin que exista control por las instituciones competentes. Por lo que es prioritario garantizar la inocuidad de las cosechas en los campos agrícolas, la cual es competencia del Senasa, que a su vez cumplen labores exclusivas en la erradicación de la mosca de la fruta, como política del gobierno en la producción de cultivos agroexportables dirigida a una agricultura consolidada en el valle de Cañete, endémica por plagas y enfermedades. En consecuencia, no existe un plan de capacitaciones o programas para la $\mathrm{AF}$, siendo de necesidad y urgencia por parte de autoridades en sanidad agraria implementar y contar con la logística indispensable.

La agricultura familiar de subsistencia en el valle de Cañete, está ligada a la producción familiar y a la consolidación de trabajo en equipo, basándose en la satisfacción de sus necesidades alimenticias del núcleo familiar, careciendo de apoyo técnico, financiero y esforzándose en actividades diversas para mejorar su economía. Destacando la ausencia de los diferentes niveles de gobierno con programas y proyectos. La incertidumbre del sector agrario en las zonas rurales, esta carente de oportunidades, siendo este una agricultura de autoconsumo, en área diseminadas por efectos demográficos y carencia de recursos económicos. La agriculura de subsistencia cuenta con escasa reserva de tierra y por consiguiente bajos ingresos que no generan una mejora de su economia, por tanto no contribuyen sustancialmente a la seguridad alimentaria ni acceso a los creditos (Acevedo, 2016a)

La agricultura consolidada, empresarial o agroexportadora que el empresario implementa para la producción nacional y mercado internacional cuenta con disponibilidad de la propiedad de la tierra saneada, tecnología, innovación, capacitaciones a su personal, entre otras. Logrando incrementar su unidad productiva, para la siembra de cultivos rentables, con financiamiento propio y privado, con garantías seguras de retorno. Permitiendo fortalecer económicamente su actividad productiva, su economía, organizar sus cultivos, mejorar la calidad de vida de su familia, generadora de puestos de trabajo, y compite en los mercados del exterior. Con solidas políticas públicas que el gobierno implementa, con fines de crecimiento laboral y oportunidades a las zonas rurales con la Población Económicamente Activa (PEA). La agricultura consolidada forma parte del bloque de agricultores con potenciales de generar recursos agropecuarios, que contribuye a incrementar sus excedentes, y les permita contar con recursos para aplicar en forma sostenible sus actividades propias de su vida productiva (Acevedo, 2016b).

\section{Discusión}

Cabe señalar que las políticas públicas están enmarcadas a desarrollar una agricultura familiar basada en la seguridad alimentaria y erradicación de la pobreza, por ello la FAO (2016)

Esta obra se comparte bajo la licencia Creative Common Atribución-No Comercial 4.0 International (CC BY-NC 4.0) Revista de la Universidad Internacional del Ecuador. URL: https://www.uide.edu.ec/ 
considera que en el mundo moderno conlleva mayor producción alimentaria en menores hectáreas agrícolas. Para el $70 \%$ de los pobres del mundo que viven en zonas rurales, la agricultura es la principal fuente de ingresos y de trabajo que se ve reflejado en el Perú. El valle de Cañete, muestra una particularidad única a las distintas regiones existentes en el Perú, en vista que cuentan con agua durante todo el año y su principal actividad es el agro, sin embargo, estas están siendo afectados por incumplimiento de las políticas públicas que impide la protección a la $\mathrm{AF}$, peligro latente para la seguridad alimentaria carente de programas y proyectos. No obstante, a ello AF trabajan unidas a la familia para el sustento diario sin apoyo de instituciones agrarias y gubernamentales; lo que significa que carecen de recursos, mercado, y tecnología, trabajando con mayor eficiencia a proteger el medio ambiente, los suelos, el agua, el aire, productos limpios de residuos de pesticidas.

Considerando que deben mejorar sus condiciones a través proyectos sostenibles y su permanencia de la AF para la sociedad, coincidiendo con Quintero (2017) quien concluye que mejorar la pequeña agricultura es combatir la pobreza rural alimentaria mediante nuevos programas con instituciones ligadas al sector, que impulsan el desarrollo agrario frente al cambio climático y lograr habilidades con distintas perspectivas tansitoria, a fin de mejorar los recursos económicos de las familias por el estado, direccionando en beneficio de las zonas rurales mas necesitadas. Es importante resaltar que las políticas públicas enmarcadas a la AF, corren el riesgo de desaparecer por el nulo apoyo de los diferentes niveles de gobierno tanto nacional, regional y local, que se ve reflejado en el mal estado de los canales de regadío, los recursos de flora y fauna sin planificación y zonificación carencia de protocolos para desastres naturales en las zonas rurales, inexistencia de seguridad jurídica (Agencia de los Estados Unidos para el Desarrollo I, 2016) sostiene que existe poca claridad sobre el marco normativo aplicable en los procesos de adjudicación de tierras, debido a la lentitud de los métodos, que causa abandonados en los tramites lo que constituye inseguridad jurídica. No obstante que no cuentan con el título de propiedad. Y que la AF esta en emergencia por el accionar de las autoridades en los distintos niveles de gobierno, por efecto de politicas dividas en los diferentes estratos de gobierno que perjudican el accionar y la estabilidad del agro que determina un accionar totalmente problemático, manifestandose en discusiones, construcciones e implementación de nuevas perspectivas en la politica para la AF (Gònzales, 2016).

El riesgo por la seguridad jurídica sobre la tierra, los propietarios de la AF no cuenta con documentos legalmente inscritos. La innovación y tecnificación agraria, carece de importancia en el valle por las instituciones responsables de mejorar la producción y productividad, esto refleja que los recursos genéticos no cuentan con la protección del Estado y menos aún por la gran mayoría de productores, por lo que Ramírez, Ruilova, \& Garzón (2015) manifiestan que la innovación es el resultado de un proceso intensivo de generación de ideas que permiten encontrar nuevas y mejores soluciones a la ya existentes, frente a la necesidad de resolver un problema, por consiguiente Minagri (2016) establece que fortalecer el Sistema Nacional de Innovación Agraria -INIA, con el apoyo y la participación del sector privado para el perfeccionamiento del sector, permitiendo que sea sostenible para las futuras generaciones, priorizando la investigación respecto a la tecnificación del riego, manejo de suelos, preservación de los recursos genéticos, que impulsen el desarrollo forestal, para lograr conservar la bioseguridad. La sanidad agraria, en el valle se encuentra en riesgo, siendo afectado por el uso irresponsable de productos tóxicos, provocando contaminación de los campos y cultivos agrícolas en consecuencia es compromiso 
de la autoridad competente promover el uso eficiente. En consecuencia, los resulados sobre la contaminaciòn de los alimentos, es preocupante y a ello se suma el hecho de que la poblaciòn no tienen un conocimiento real del grado de contmiancion de algunos productos agricolas, caso del tomate, fresa y restos de antibioticos en carnes de animales (Delgado, Alvarez, \& Yañez, 2018)

La prioridad de desarrollar las capacidades de trabajo agrícola, con la intensión de mejorar la sanidad agraria de los cultivos del Valle de Cañete, a todos los productores en su conjunto màs no solo a los grupos organizados en asociaciones y cooperativas, que a su vez están integrados por pequeños agricultores; sin embargo, prima la desconfianza entre ellos, reflejo de una reforma agraria insostenible. Y logrando que las empresas privadas de ventas insumos agrícolas (pesticidas y fertilizantes sintéticos) ingresen a capacitar a los productores en forma general, favoreciendo sus intereses. Por consiguiente, el desarrollo de capacidades, como la innovación y la mejora de conocimiento en las personas, los dirigentes, los líderes de las organizaciones y la sociedad, conllevan un cambio guiado y sostenido de capacidades. (Programa de las Naciones Unidas para el Desarrollo, 2009). En el acceso a mercados se fundamenta con la información eficiente y oportuna que deben contar los productores de campo para efectuar la siembra y comercialización, basados en reportes oficiales que contribuya a mejorar sus ventas. En tanto que las relaciones de reciprocidad consolidan las redes sociales que facilita el acceso a los mercados... (Sabourin, et. al., 2018).

Finalmente, la agricultura consolidada efectúa su crecimiento en basa a su propio esfuerzo y con el apoyo de políticas claras definidas por el gobierno, basándose en una agricultura agro exportadora, con fuentes de trabajos para jóvenes entre hombres y mujeres, adultos de diferente género, identificado cada uno de ellos las actividades precisas según responsabilidad. Y las polìticas pùblicas no siguen "recetas" por lo que no pueden ser consideradas procesos amorfos, desconectados de la realidad social (Flexor \& Grisa, 2016, p. $51)$.

\section{Conclusiones}

Las políticas en la agricultura familiar en el valle de Cañete, existe desconocimiento de la ley, tanto de autoridades y productores. Por consiguiente, se ve reflejado en la mala distribución del agua de regadío, por canales sin revestimiento, provocando erosión, infiltración, desperdicio, lavado de suelos y estos a su vez la salinización, enfermedades fungosas y desnutrición, como también por ausencia de sistemas de riegos tecnificados. La Disminución de la flora y la preservación de la fauna en los campos AF, a diferencia de la AC, cuenta con mayor presencia de flora en los límites de sus áreas agrícolas y fauna controlada, por la regulación de organismos competentes internacionales, lo que viene provocando insostenibilidad, y desequilibrio del ecosistema y la biodiversidad agrícola en el valle. También existe un riesgo latente por la seguridad jurídica sobre la tierra en familias dedicadas al campo, por carecer de iniciativa del gobierno en las políticas públicas de formalización, respecto a la $\mathrm{AF}$, más no a una AC. El financiamiento agrario en la AF no es viable debido a que no cuentan con garantías, debido a que vienen laborando con recursos propios limitados en pequeña escala y la diversidad de actividades que generan para su subsistencia. Sin embargo, la AC está marcada a una serie de iniciativas propias y financieras privadas. En el seguro agrario, existe solo para efectos de salud, emitida por la institución competente de la agencia agraria por medio de una constancia, sin embargo, el 
seguro agrario por condiciones climáticas no está considerada. La Innovación y tecnificación agraria, está ausente por parte de la entidad estatal, claro reflejo de una política centralista en descuido de la AF. Reportando iniciativa en la AC. En la gestión de riesgo, hay una débil existencia e inoperancia en la articulación de las instituciones locales, regionales y nacionales por carecer de planificación, ocasionando pérdidas económicas por gestiones inoportunas, provocando vulnerabilidad en el valle por efectos de cambio climático. También hay una ausencia de articulación al mercado a los de la AF. En tanto, la sanidad agraria en el valle de Cañete, cuenta con una sola política para erradicar la mosca de la fruta por parte de Senasa, orientando a mejorar la calidad de las frutas de exportación más no el uso indiscriminado de plaguicidas en los demás cultivos, perjudicando a los consumidores por el consumo de alimentos con residuos de pesticidas (falta de inocuidad para el consumo local).

La agricultura de subsistencia en el valle de Cañete, esta privada de recursos económicos, y tecnológicos, efecto de ello solo produce para el autoconsumo, con débil o nulo mercado, permite conservar el recurso suelo y la biodiversidad. La producción familiar se basa en el trabajo en forma conjunta que desarrolla la familia en horas de campo, y su diversificación monetaria se basa en otras actividades según oportunidades que se le presentan. El auge del crecimiento poblacional refleja el auge del crecimiento de la agricultura de subsistencia, en pequeñas extensiones agrícolas que genera el valle, producto de la venta de sus predios. Por tanto, aumenta el índice de la pobreza rural y urbana que paulatinamente están siendo absorbidas por el crecimiento de la población, en especial de aquellas familias beneficiadas por la reforma agraria. En consecuencia, la seguridad alimentaria está en riesgo por la inactividad de las políticas públicas propuestas para la agricultura familiar en desventaja comparativa de la agricultura agroexportadora.

La agricultura consolidada en el valle de Cañete se ve reflejada mediante el desarrollo del campo, de cultivos de agro exportación generadora de divisas, que labora mediante sistemas de calidad internacional, captando mano de obra de los miembros dedicados a la AF, estudiantes, amas de casa, jóvenes entre hombres y mujeres, logrando muchas veces la elevada competencia por recursos humanos en campañas de cosechas entre empresas. La AC cuenta con tecnología propia de maquinarias, equipos, mercados, capacitaciones e investigación, expresando la competitividad en el mundo agrario. Esta modalidad es generalizada en el valle, por unidades productivas en crecimiento, por iniciativa del empresario y la estructura de un sistema de derecho a la propiedad que genera confianza y estabilidad protegida por leyes del gobierno.

\section{Bibliografía}

Acevedo Osorio, A. (2016). Contribuciones y retos de la agricultura familiar en Colombia. Colombia: Universidad Cooperativa de Colombia. doi:org/10.16925/9789587600476.

Agencia de los Estados Unidos para el Desarrollo I. (2016). Seguridad juridica en la tenencia de la tierra en la iniciativa para la conservaciòn en la amazonìa andina. IICA.

Balestro, M., Sayago, D., Valencia, M., \& Sabourin, E. P. (2019). Difusiòn regional de polìticas pùblias de desarrollo rural. Mundo Plural, Revista Latinoamericana de Políticas y Acción Pública, 5(2), 1. doi:10.17141/mundosplurales.2.2018.3374

$\begin{array}{cccc}\text { Banco } & \text { Interamericano } & \text { de } & \text { Desarrollo. } \\ & \text { https://webimages.iadb.org/publications/spanish/document/Pautas-para-la- }\end{array}$

Esta obra se comparte bajo la licencia Creative Common Atribución-No Comercial 4.0 International (CC BY-NC 4.0) Revista de la Universidad Internacional del Ecuador. URL: https://www.uide.edu.ec/ 
elaboraci\%C3\%B3n-de-estudios-de-caso.pdf. (BID,

Editor)

doi:https://webimages.iadb.org/publications/spanish/document/Pautas-para-laelaboraci\%C3\%B3n-de-estudios-de-caso.pdf

Centro Nacional de Planeamiento Estratégico. (2011). Plan Bicentenario, El Perù hacia el 2021. Lima.

Douwe van der Ploeg, J. (2013). Agricultura familiar campesina. 6.

Echeverri P., A., \& Renault A., A. (2017). Guìa para la generaciòn participativa de polìticas pùblicas diferenciadas para la agricultura familiar. Instituto Interamericano de Cooperaciòn para la Agricultura IICA, 17. doi:org/10.1787/9789264257108-en.

Flexor, G., \& Grisa, C. (2016). Politicas de seguridad alimentaria y agricultura familiar en Brasil: Actores, ideas e instituciones. América Latina Hoy, 74, 51. doi:10.14201/alh2016743953

Frei Ruiz-Tagle, E. (1997). Gobernabilidad democràtica. Chile: Andres Bello.

Gònzales, F. (2016). Politias para la agricultura familiar: Transformaciones territoriales en el sudoeste Bonaerense(Tesis doctoral). Buenos Aires. Obtenido de http://repositorio.filo.uba.ar/handle/filodigital/3183

Luna Hèrnandez, , S. M., \& Maldroñero Palacios, S. M. (2016). Importancia del componente social en el manejo de recursos hìdricos. Luna Azul(42), 12. doi:10.17151/luaz.2016.42.13

Maletta, H. (2017). La pequeña agricultura familiar en el Peru. Una tipologia microrregionalizada (5 ed.). Lima: FAO. Obtenido de http://www.fao.org/3/a-i6759s.pdf

Martìnez Carazo, P. C. (2006). El mètodo de estudio de caso. Estrategia metodològica de la investigaciòn cientifica. Colombia.

Ministerio de Agricultura y Riego. (2015). Polìtica Nacional Agraria. 34.

Ministerio de Agricultura y Riego. (2016). Decreto Supremo que aprueba la Polìtica Nacional Agraria. pág. 5. Obtenido de http://www.minagri.gob.pe

Organización de las Naciones Unidas para la Agricu. (2016). Agricultura y desarrollo rural. Obtenido de https://datos.bancomundial.org/indicador/NV.AGR.TOTL.ZS

Programa de las Naciones Unidas para el Desarrollo. (2009). Desarrollo de capacidades. UNDP, 4. Obtenido de https://www.undp.org/content/dam/undp/library/capacitydevelopment/spanish/Capacity_Development_A_UNDP_Primer_Spanish.pdf

Quintero Peralta, M. A. (2017). Politicas pùblicas, soberanìa alimentaria y estategias campesinas en zonas rurales pobres de Mèxico(Tesis doctoral). Còrdoba, España: UCOPress. Obtenido de http://www.uco.es/servicios/ucopress/index.php/es/

Ramìrez Morales, I., Ruilova Reyes, B., \& Garzòn Montealegre, J. (2015). Innovaciòn tecnològia en el sector agropecuario. Machala, Ecuador: Universidad Tècnica de Machala. Obtenido de http://repositorio.utmachala.edu.ec/bitstream/4800

Ramos, A. (2016). Definiciòn de "Agricultura familiar" como categorìa socioeconòmica. 21. doi:org/10.17058/redes.v21i3.8243

Rico y Lorenzo, C. (2006). Contribuciòn sobre los paradigmas de investigaciòn. Dialnet, 31(01). doi:10.5902/198464441486

Sabourin, E., Aveline, I., Petersen, P., \& Para, M. (2018). Construcciòn soial del acceso a los mercados por agricultores. Politica Econòmica y Desarrollo Sostenible, 3(1). doi:org/10.15359/peds.3-1.1

Salcedo, S., \& Guzmàn, L. (2014). Agricutura familiar en Amèrica Latina y el Caribe. 404. doi:10.1787/agr_outlook-2013-es.

Esta obra se comparte bajo la licencia Creative Common Atribución-No Comercial 4.0 International (CC BY-NC 4.0) 
Torres- Melo, J., \& Santander A., J. (2013). Introducciòn a las polìticas pùblicas. Colombia: IEMP.

Troncoso-Pantoja, C., \& Amaya-Placencia, A. (2016). Entrevista: guìa pràctica para la recolecciòn de datos cualitativos en investigaciòn de salud. Revista de la Facultad de Medicina, 65(2). doi:10.15446/revfacmed.v65n2.60235

Villarreal, F. (2018). La inclusiòn de la agricultura familiar. Discusiòn de su uso en programas de desarrollo rural en Argentina. Mundo Agrario, 19(41), 10. doi:org/10.24215/15155994e091 